
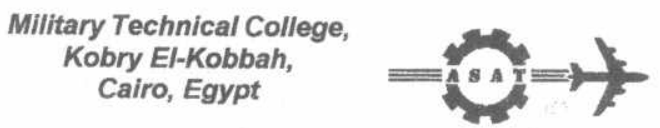

$9^{\text {th }}$ International Conference

On Aerospace Sciences \& Aviation Technology

\title{
SOFTWARE PACKAGE FOR CALCULATION AND PLOTTING OF THE SPOT DIAGRAM AND POINT SPREAD FUNCTION OF AN OPTICAL ITEM USED IN AN INFRARED SEEKER
}

\author{
Elfarouk* A. O., Saad* S. M., Ali* I. A.
}

\begin{abstract}
Any optical item (lens or mirror) is characterized by its spot diagram and point spread function. This paper is directed toward constructing a software package for the calculation and plotting of the spot diagram and point spread function. It can be used as a tutorial or a procedure for the design of an optical system, which can be used in building up a seeker head (homing head).
\end{abstract}

\section{KEY WORDS}

Paraxial ray tracing, meridianal ray tracing, spot diagram, point spread function

\section{INTRODUCTION}

The analysis of an optical system requires a great deal of numerical computation devoted, for the most part, to the determination of the exact paths taken by light rays as they pass through the system. A ray may be traced by the application of Snell's law at each surface. Today the most widely used tool for ray tracing is the computer. They are further characterized by the fact that the quantities involved in them are "bounded", that is, the maximum size of each term of an equation is readily predicted in terms of the size of the optical system.

Each set of ray tracing equations will be presented in four operational sections. First, the "opening" equations which start the ray into the system; second, the "refraction" equations which determine the ray direction after passing through a surface; third, the "transfer' equations which carry the computation to the next surface; and fourth, the "closing" equations which permit the determination of the final intercept length or height.

\footnotetext{
* Egyptian Armed Forces
} 
The "refraction" and "transfer" equations are used iteratively, that is, they are repeated for each surface of the system. The "opening" and "closing" equations are only used at the start and finish of the computation. $[1,3]$

\section{PARAXIAL RAY TRACING}

Opening: Given $Y$ and $u$ at the first surface

$$
Y=10
$$

Or

$\mathrm{Y}=\mathrm{h}+\mathrm{su}$

Refraction:

$u^{\prime}=\frac{c Y\left(N^{\prime}-N\right)}{N^{\prime}}+\frac{N u}{N^{\prime}}$

Transfer to the next surface:

$Y_{2}=Y_{1}-t_{1}^{\prime}$

$\mathrm{u}_{2}=\mathrm{u}_{1}^{\prime}$

Closing:

$\mathrm{l}^{\prime}=\frac{\mathrm{Y}}{\mathrm{u}^{\prime}}$

Or

$h^{\prime}=Y-s^{\prime} u^{\prime}$

The symbols have the following meanings:

$Y$ the height at which the ray strikes the surface; positive above the axis, negative below.

$Y_{1} \quad$ the height at which the ray strikes the first surface.

$Y_{2}$ the height at which the ray strikes the second surface

$u$ the slope of the ray before refraction

$u^{\prime} \quad$ the slope of the ray after refraction; ray slopes are positive if the ray must be moved counterclockwise to reach the axis.

$\mathrm{h}$ the height in the object plane at which the ray originates; sign convention same as $y$.

h' the height at which the ray intersects the image plane.

I the distance from the first surface of the system to the axial intercept of the ray; negative if intercept point is to the left of the last surface.

I. the distance from the last surface to the final axial intercept of the ray; positive if the intercept is to the right of the last surface.

$\mathrm{s} \quad$ the distance from the first surface to the image plane; negative if the object plane is to the left of the surface.

$s^{\prime} \quad$ the distance from the last surface to the image plane; positive if the image plane is to the right of the surface. 
the curvature (reciprocal radius) of the surface equal to $1 / 2$; positive if the center of the curvature is to the right of the surface.

$\mathrm{N}$ the index of refraction preceding the surface

$\mathrm{N}^{\prime}$ the index of refraction following the surface

$t$ the vertex spacing between surfaces $K$ and $K+1$

$\mathrm{N}, \mathrm{N}$ 'and $\mathrm{t}$ are positive when the ray travels from left to right, negative when the ray travels from right to left (as it does following a reflection)

The physical meanings of the symbols are indicated in Fig. (1)
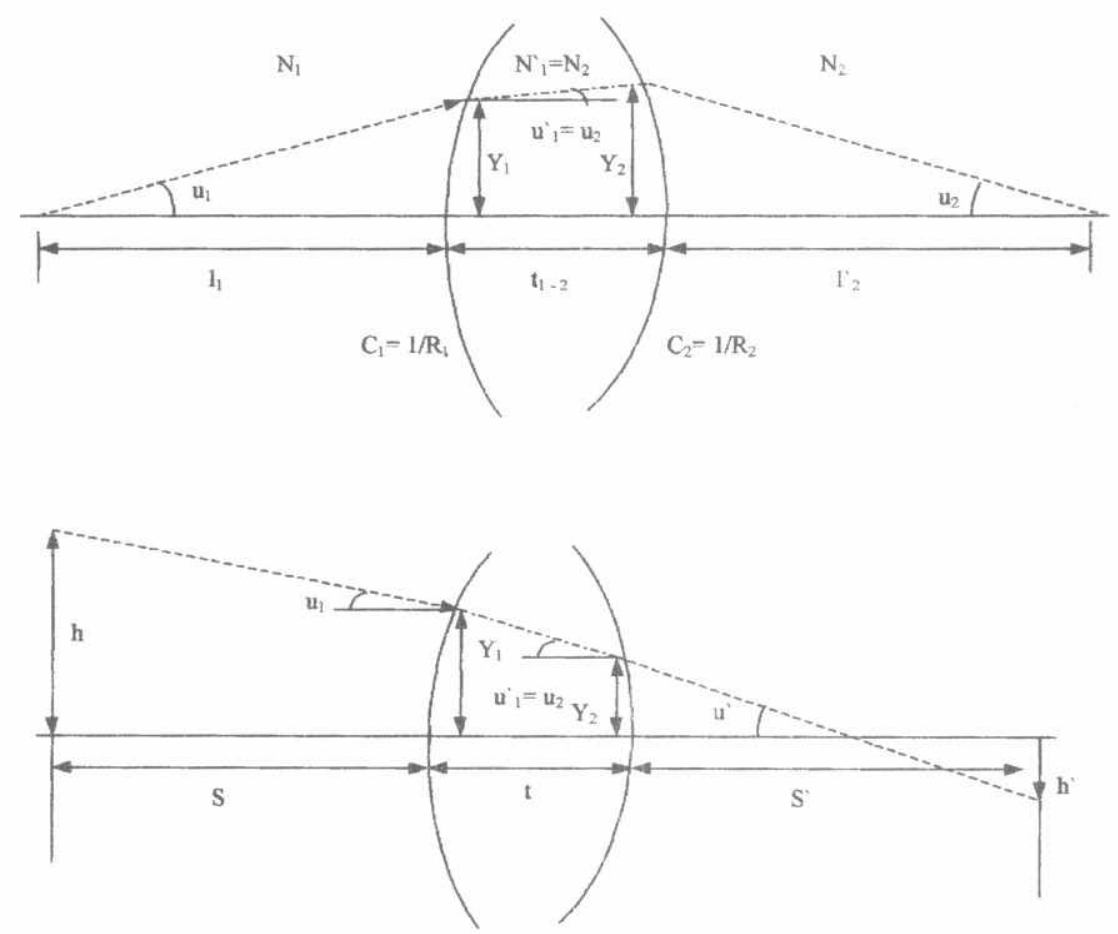

Fig 1 Diagrams to illustrate the symbols used in the paraxial ray tracing equation 


\section{MERIDIONAL RAY TRACING}

Meridional rays are those rays, which are co-planar with the optical axis of the system. The plane in which both ray and axis lie is called the meridional (accent on the first i) plane and, in an axially symmetrical system, a meridional ray remains in this plane as it passes through the system. The two dimensional nature of the meridional ray makes it relatively easy to trace. For great many optical systems, the information gained from tracing a few meridional rays are sufficient for most purposes. [4,5]

Opening Given $Q$ and $\sin U$ at the first surface.

$\mathrm{Q}=\mathrm{L} \sin \mathrm{U}$

Or

$\mathrm{Q}=\mathrm{H} \cos \mathrm{U}+\mathrm{s} \sin \mathrm{U}$

Refraction:

$\sin \mathrm{I}=\mathrm{Qc}-\sin \mathrm{U}$

$\sin \mathrm{I}^{\prime}=\frac{\mathrm{N} \sin \mathrm{I}}{\mathrm{N}^{\prime}}$

$\cos \mathrm{I}^{\prime}=\sqrt{1-\sin ^{2} \mathrm{I}^{\prime}}$

$\sin U^{\prime}=\sin (U+I) \cos I^{\prime}-\cos (U+I) \sin I^{\prime}$

$\cos U^{\prime}=\cos (U+I) \cos I^{\prime}+\sin (U+I) \sin I$

$Q^{\prime}=\frac{Q\left(\cos U^{\prime}+\cos I^{\prime}\right)}{(\cos U+\cos I)}$

Transfer:

$\mathrm{Q}_{2}=\mathrm{Q}^{\prime},-\mathrm{t} \sin \mathrm{U}^{\prime}$

$\mathrm{U}_{2}=\mathrm{U}^{\prime}$

Or

$H^{\prime}=\frac{Q^{\prime}-s^{\prime} \sin U^{\prime}}{\cos U^{\prime}}$ 
Miscellaneous:

$$
\begin{aligned}
& \mathrm{y}=\frac{\mathrm{Q}[1+\cos (\mathrm{U}+\mathrm{I})]}{(\cos \mathrm{U}+\cos \mathrm{I})}=\frac{\mathrm{Q}^{\prime}[1+\cos (\mathrm{U}+\mathrm{I})]}{\left(\cos \mathrm{U}^{\prime}+\cos \mathrm{I}^{\prime}\right)} \\
& \mathrm{x}=\frac{\mathrm{Q} \sin (\mathrm{U}+\mathrm{I})}{(\cos \mathrm{U}+\cos \mathrm{I})} \\
& \mathrm{D}_{\mathrm{1} 1 \mathrm{2} 2}=\frac{\mathrm{t}-\mathrm{x}_{1}+\mathrm{x}_{2}}{\cos \mathrm{U}_{1}^{\prime}}
\end{aligned}
$$

Where

$Q$ the distance from the vertex of the surface to the incident ray, perpendicular to the ray; positive if upward.

$Q^{\prime} \quad$ the distance from the surface vertex to the refracted ray, perpendicular to the ray

1 the angle of incidence at the surface; positive if the ray must be rotated clockwise to reach the surface normal (i.e. the radius)

I' the angle of refraction

$x$ the longitudinal coordinate (abscissa) of the intersection of the ray with the surface; positive if the intersection is to the right of the vertex.

$D_{1 \text { to2 }}$ the distance along the ray between surface 1 and surface 2 The physical meanings of the symbols are indicated in Fig. (2)

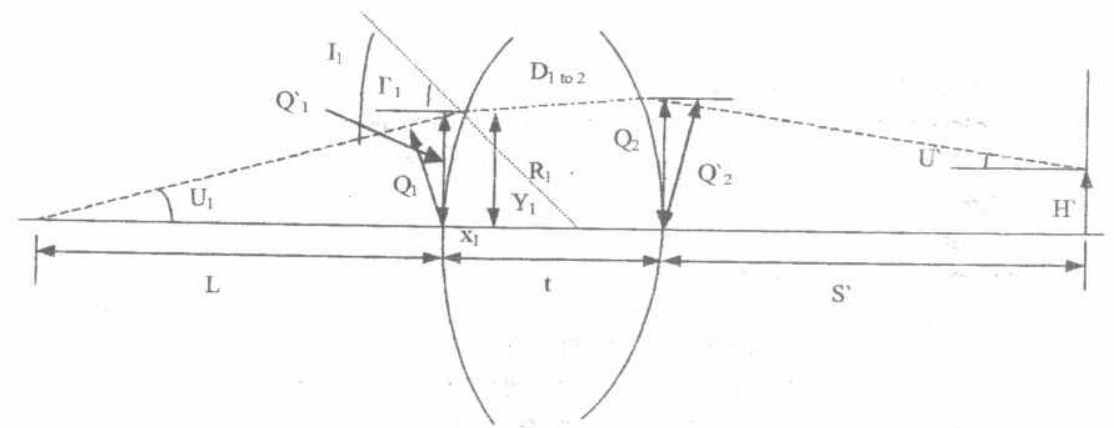

Fig 2 Diagrams to illustrate the symbols used in the meridional ray tracing equation 


\section{MODELING OF AN OPTICAL ITEM PERFORMANCE}

A software package is developed using a computer programming language, Borland $\mathrm{C}++[6]$, for modeling the optical item of a typical Infrared seeker head. The idea of this software package is to calculate and plot the spot diagram and point spread function to achieve the performang number of steps. These steps item when it is used in an

are carried out as follows: First, we perform a paraxial ray tracing thint the place of the image plane for a posed on considering a small angle element. The paraxial ray tracing is based (paraxial ray).

(paraxial angle) less than $1^{\circ}$ for the incidentrormed to calculate the position and Second, a meridional ray tracing is

height of the image at the image plane. job for the rays passing through the Third, after finishing the ray-tracing will have well defined images (strikes) at aperture of the optical element, we will have well dere is a symmetry between the image plane. Taking into consideration that we will rotate these points in one the quarters of the optical element (lens), wive so called spot diagram of the quarter then, multiplying by four will give us the so called spot dagram of thead function of optical item. Finally, through the spot is achieved by counting the number of spots between the optical element, this is ach the lines bounding an assumed increr of points against $x$. be the normalized plot of the number for points of the new software packe In the following section, a complete flowchart of the

\section{ALGORITHM FOR CALCULATING AND PLOTTING
DIAGRAM AND THE POINT SPREAD FUNCTION}

- Paraxial ray tracing

- Calculation of the height at which the ray strikes the $1^{\text {st }}$ surface (y1)

height at which the ray strikes the $2^{\text {nd }}$ surface $(y 2)$

- Calculation of the height at whe ray before refraction (u)

- Calculation of the slop of the ray before final axial

- Calculation of the dista

Meridional ray tracing

vertex of the $1^{\text {st }}$ surface to the

- Calculation of the distance to ray $(Q)$

incident ray perpendicular the angle of incidence at the $1^{\text {st }}$ surface (I)

- Calculation of the angle of refraction ( $\left.I^{\prime}\right)$

- Calculation of the slop of the ray after refraction $\left(\mathrm{U}^{*}\right)$ 
- Calculation of the distance from the $2^{\text {nd }}$ surface vertex to the refracted ray $\left(Q^{\prime}\right)$

- Calculation of the distance from the last surface to the final axial intercept of the meridional ray (L')

- Calculation of the height of the image at the image plane.

- Plotting of the spot diagram

- Calculation of the height of the image at the image plane for each ray.

- Rotating each point round the axes of the image plane.

- Calculation and plotting of the point spread function

- Counting the number of points in the spot diagram within a certain $\Delta x$.

- Plotting of the number of points $\mathrm{Nx}$ against $\mathrm{X}$.

\section{OUTPUT OF THE SOFTWARE PACKAGE}

\section{A LENS:}

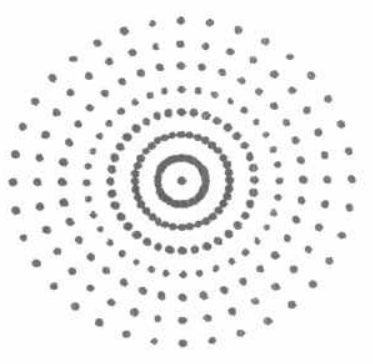

Fig. 3 Spot diagram of a lens

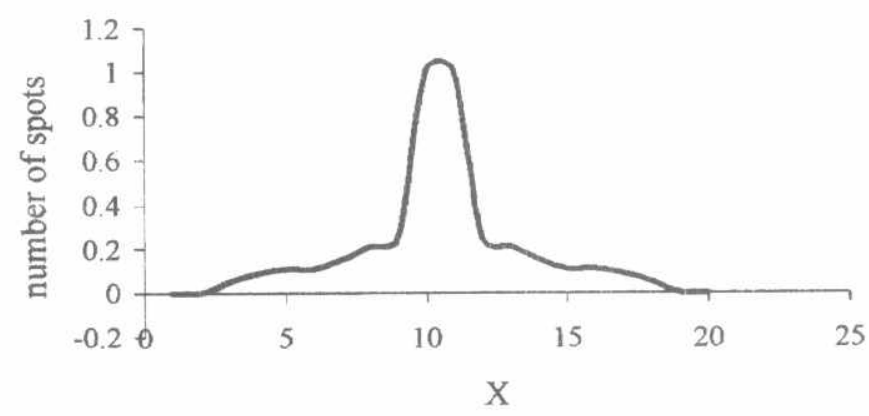

Fig. 4 point spread function of a lens 


\section{A MIRROR:}

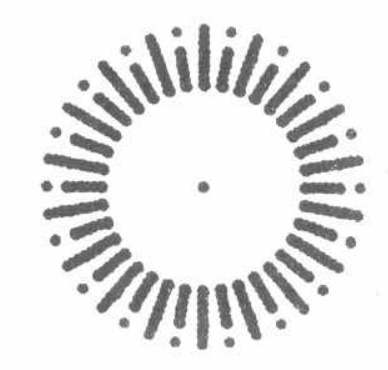

Fig. 5 Spot diagram of a mirror

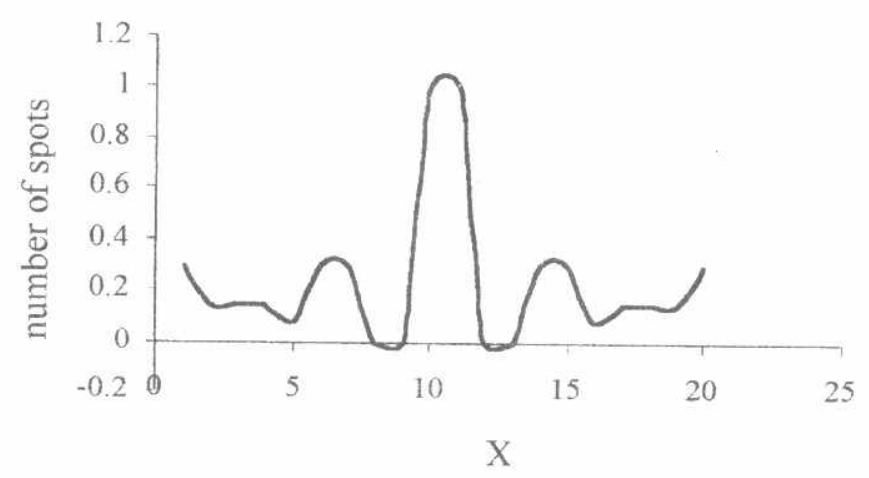

Fig. 6 point spread function of a mirror

Where:

$X$ is the width of the window which the number of the points counted within 


\section{CONCLUSION}

In order to model the performance of an optical item studying its behavior in several conditions is needed. Obtaining the spot diagram and the point spread function of the item enables that study. The results of the new software package will allow the designer to determine the best dimensions and shape of the optical item, which will give excellent performance and hence minimum aberrations. We hope that this paper will facilitate and enhance the accuracy of modeling the performance of the optical system used in the IR seeker.

\section{REFERENCES}

[1] R.Kingslake, "Optical System Design", Academic Press Inc., New York, 1983.

[2] D.C.O'Shea,"Elements of Modern Optical Design", Joh Wiley, 1985.

[3] Warren J. Smith "Modern Optical Engineering", New York, 1966.

[4] D.Malacara, Z.Malacara,"Handbook of Lens Design", Marcel Dekker Inc., New York, 1994.

[5] R.Kingslake,"Lens Design Fundamentals", Academic Press Inc., New York, 1978.

[6] "C: The Complete Reference", $2^{\text {nd }}$ Edition, McGraw-Hill Inc., 1990.

[7] "Borland $\mathrm{C}^{++}$, Ver3.1", Borland International Inc., 1992. 